\title{
Retailer's inventory system in a two-level trade credit financing with selling price discount and partial order cancelations
}

\author{
A. Thangam
}

Received: 12 September 2013/Accepted: 17 December 2013/Published online: 11 March 2015

(C) The Author(s) 2014. This article is published with open access at Springerlink.com

\begin{abstract}
In today's fast marketing over the Internet or online, many retailers want to trade at the same time and change their marketing strategy to attract more customers. Some of the customers may decide to cancel their orders partially with a retailer due to various reasons such as increase in customer's waiting time, loss of customer's goodwill on retailer's business, attractive promotional schemes offered by other retailers etc. Even though there is a lag in trading and order cancelation, this paper attempts to develop the retailer's inventory model with the effect of order cancelations during advance sales period. The retailer announces a price discount program during advance sales period to promote his sales and also he offers trade credit financing during the sales periods. The retailer availing trade credit period from his supplier offers a permissible delay period to his customers. The customer who gets an item has allowed paying on or before the permissible delay period which is accounted from the buying time rather than the start period of inventory sales. This accounts for significant changes in the calculations of interest payable and interest earned by the retailer. The retailer's total cost is minimized so as to find out the optimal replenishment cycle time and price discount policies through a solution procedure. The results derived in mathematical theorems are implemented in numerical examples and sensitivity analyses on several inventory parameters are obtained.
\end{abstract}

Keywords Inventory - Advance sales - Price discount . Two-echelon trade credit

\footnotetext{
A. Thangam $(\bowtie)$

Department of Mathematics, Pondicherry University, Community College, Lawspet-08, 605008 Pondicherry, India e-mail: thangamgri@yahoo.com
}

\section{Introduction}

In today's business era, retailers have the dominant power of controlling or affecting another member's decision in a supply chain. A retailer has the ability to offer an effective promotional effect such as price discount, credit period etc. The retailers often try to stimulate the demand by offering price discounts. Price discounts could improve economic benefits to consumers and influence consumers' beliefs about the brand which will increase consumers' purchase intentions. In real life, there are situations in which the retailer announces price discount offers to the customers who can commit their orders before the selling period. Due to the booming in IT, customers can easily commit their orders prior to selling period and the estimation error in demand can also be reduced. This situation is adopted more in the selling of musical disks, apparel, video games, or books. In dairy product manufacturing scheme prior to selling season, the retailer offers price discount to the customers who register their orders via email or phone call etc.

Certainly, the credit period facility would promote the purchases and it attracts new customers who consider trade credit policy as a type of price reduction. To handle the risks of trade credit situations, retailer collects a higher interest from his customers when they did not settle the payment within the credit period time. In this paper, supplier offers the retailer a trade credit period $t_{1}$. The retailer offers its customers a credit period $t_{2}$ and he receives the revenue from $t_{2}$ to $T+\mathrm{t}_{2}$, where $T$ is the cycle time at the retailer. Under this situation, three cases such as $t_{1} \leq T$, $T \leq t_{1} \leq T+t_{2}$ and $T+t_{2} \leq t_{1}$ are to be considered. Customers under advance sales booking system may cancel their reservations due to various reasons such as increase in customer's waiting time, loss of customer's goodwill on 
retailer's business, attractive promotional schemes offered by other retailers etc. Here, we consider the partial order cancelations during advance sales period.

This paper investigates retailer inventory system in which customers are partially canceling their orders during advance sales period. The customers commit their orders before the selling period. Among the committed orders, a fraction of orders are canceled. The customer who receives an item at time ' $t$ ' will remit at time $t+t_{2}$ due to his availability of trade credit period $t_{2}$. So, the retailer gets revenue from earning the interest on customer's payment during the period from $t_{2}$ to $t_{1}$ and he pays interest during the period $t_{2}$ to $T+t_{2}$. So, the cases in Tsao (2009) are to be reconsidered as $t_{1} \leq T, T \leq t_{1} \leq T+t_{2}$, and $T+t_{2} \leq t_{1}$. The retailer also earns interest from stand-by orders during advance sales period. During the normal sales period, all customers receive their orders at the time of their purchase. With the help of derived mathematical theorems in the model, a simple solution procedure is provided to find the optimal solution. Numerical examples are given to illustrate the solution process and sensitivity analyses are performed for various inventory key parameters.

\section{Literature review}

During the past few years, many researchers have studied inventory models for permissible delay in payments. Goyal (1985) was the first proponent for developing an economic order quantity (EOQ) model under the conditions of permissible delay in payments. Shah (1993) considered a stochastic inventory model when items in inventory deteriorate and delays in payments are permissible. Aggarwal and Jaggi (1995) extended Goyal's (1985) model to allow the inventory to have deteriorating items. Jamal et al. (1997) further generalized Aggarwal and Jaggi's (1995) model to allow for shortages. Hwang and Shinn (1997) developed a model considering exponentially deteriorating items and found decision policy for selling price and lot size. Teng (2002) amended Goyal's (1985) model by considering the difference between unit price and unit cost and established an easy analytical closed-form solution to the problem. Chang et al. (2003) constructed a mathematical model for an EOQ inventory with deteriorating items and supplier credits are linked to ordering quantity. Chung and Huang (2003) generalized Goyal's (1985) EOQ model to an economic production quantity (EPQ) model in which the selling price is the same as the purchase cost. Huang (2003) extended Goyal's (1985) model to the case in which the supplier offers the retailer the permissible delay period $M$ (i.e., the up-stream trade credit), and the retailer in turn provides the trade credit period $N$ (with $N<M$ ) to its customers (i.e., the down-stream trade credit). Ouyang et al. (2006) developed an EOQ model for deteriorating items under trade credits. Teng and Goyal (2007) amended Huang's (2003) model by complementing his shortcomings. Liao (2007) established an EPQ model for deteriorating items under permissible delay in payments. Chang et al. (2008) reviewed the contributions on the literature in modeling of inventory lot-sizing under trade credits. Ho et al. (2008) developed an integrated supplier-buyer inventory model with the assumption that demand is sensitive to retail price and the supplier adopts a two-part trade credit policy. Huang and Hsu (2008) have developed an inventory model under two-level trade credit policy by incorporating partial trade credit option at the customers of the retailer. Liao (2008) developed an EOQ model with non-instantaneous receipt and exponentially deteriorating items under two-level trade credit financing. Teng and Chang (2009) extended the Huang (2007) model by relaxing the assumption $N<M$. Jaggi et al. (2008) developed a simple EOQ model in which the retailer's demand is linked to credit period. Thangam and Uthayakumar (2009) developed an EPQ model for perishable items under twolevel trade credit policy when demand depends on selling price and credit period. Teng (2009) developed an EOQ model for a retailer who receives a full trade credit from its supplier and offers a partial trade credit to its bad credit customers or a full trade credit to its good credit customers. Teng et al. (2009) developed a mathematical model for an EOQ inventory with two warehouses and solved the problem by an arithmetic-geometric in-equality method. Tsao (2009) developed a model by considering advance sales discount and trade credits. In Tsao's (2009) paper, he considered a strategy, namely advance sales discount (ASD) program that the customers can commit their orders at a discount price prior to the selling season. He considers the cases such as $T \geq t_{1}, t_{2} \leq T \leq t_{1}$ and $T \leq t_{2}$. Chen and Kang (2010a) considered trade credit and imperfect quality in an integrated vendor-buyer supply chain model. Concurrently, Chen and Kang (2010b) developed integrated vendor-buyer inventory model with two-level trade credits and price negotiation scheme. Chang et al. (2010) have extended the Liao (2008) model by considering the case $M<N$ also. Hu and Liu (2010) established an EPQ model with permissible delay in payments and allowable shortages. Cárdenas-Barrón et al. (2010) developed a model which considers the advantage of a one-time discount offer with allowed backorders. Balkhi (2011) has developed a finite horizon inventory model with deteriorating items under inflation and time value of money when shortages are not allowed. Thangam and Uthayakumar (2011) have built a mathematical model for a retailer under two-level trade credit and two-payment methods. Tsao (2011) developed an EOQ model by considering trade credit and logistics risk. Teng et al. (2011) extended an EOQ model for stock-dependent demand to supplier's trade credit with 
a progressive payment scheme. Skouri et al. (2011) studied supply chain models for deteriorating items with ramp-type demand rate under permissible delay in payments. Jaggi et al. (2012) developed an EOQ model under two levels of trade credit policy when demand is influenced by credit period. Liao et al. (2012) have developed a two warehouse lot-sizing model with order dependent trade credit period. Tsao and Sheen (2012) have developed a multi-item supply chain model with trade credit periods and weight freight cost. Thangam (2012) developed a two-level trade credit financing model for a supply chain with deteriorating items and advance payment scheme. Teng et al. (2012a) developed vendor-buyer inventory models with trade credit financing under a non-cooperative and an integrated environments. Concurrently, Teng et al. (2012b) proposed an EOQ model with trade credit financing for increasing demand. Min et al. (2012) established an EPQ model with inventory-level-dependent demand and permissible delay in payments. Tsao (2012) considered manufacturer's production and warranty decisions for an imperfect production system under system maintenance and trade credit. Teng et al. (2013) have developed a two-level trade credit financing model with timely increasing demand at the retailer. Feng et al. (2013) have developed an EPQ inventory model with supplier's cash discount and twolevel trade credit financing. Ouyang et al. (2013) have developed a mathematical model with two-level trade credit financing in which trade credit offer depends on the amount of ordering quantity. Taleizadeh et al. (2013) have developed an EOQ model with perishable items, special sale offers, and shortages. Chung and Cárdenas-Barrón (2013) presented a simplified solution procedure to an EOQ model for deteriorating items by Min et al. (2010) with stock-dependent demand and two-level trade credit. Chern et al. (2013) established Stackelberg solution in a vendorbuyer supply chain model with permissible delay in payments. Ouyang and Chang (2013) proposed an optimal production lot with imperfect production process under permissible delay in payments and complete backlogging. Chen et al. (2013a) established the retailer's optimal EOQ when the supplier offers conditionally permissible delay in payments link to order quantity. Concurrently, Chen et al. (2013b) attempted to overcome some shortcomings of mathematical model and expressions in Liao et al. (2012). Jaggi et al. (2013) established an EOQ inventory model with defective items under allowable shortages and trade credit.

\section{Mathematical model formulation}

We follow the same notations as in Tsao (2009) and introduce a new notation, namely $\delta$ (fixed) referring the rate at which the orders are canceled during advance sales period.

$p$ Unit retailer price,

$c$ unit purchase cost,

$A$ ordering cost per order,

$H$ unit inventory holding cost,

$t_{1}$ retailer's credit period provided by supplier,

$t_{2}$ customer's credit period provided by retailer,

$r$ price discount,

$\delta$ the rate at which the orders are canceled during advance sales period,

$I_{\mathrm{p}}$ The interest paid per dollar per unit time,

$I_{\mathrm{e}}$ The interest earned per dollar per unit time,

$T$ Replenishment cycle time,

$D_{1}$ Annual demand rate for the retailer, say retailer 1, to whom optimum decision policy is considered,

$D_{2}$ annual demand rate for other retailers,

$Y_{1}$ fraction of the retailer 1's customers who use advance sales discount program,

$Y_{2}$ Fraction of other retailers' customers who use advance sales discount program,

$\left(1-Y_{1}\right) D_{1}$ Annual demand of customers who are not using advance sales discount program.

We follow the assumptions as in Tsao (2009) and include other assumptions that:

1. The orders during advance sales period are partially canceled.

2. The customer who gets an item at time $t$ pays at time $t+t_{2}$ and so the retailer earns interest from the revenue obtained during the time $t_{2}$ to $t_{1}$ instead of time $t=0$ to $t=t_{1}$. Retailer starts paying interest for the items in stock at the rate $I_{\mathrm{p}}$

Assumptions as in Tsao (2009):

1. The problem considers an inventory system with single item

2. The retailer offers price discount $r$ to his customers if they can commit their orders prior to the sales period.

3. $Y_{1}$ percentage of the retailer 1's customers use advance sales discount program and $Y_{2}$ percentage of other retailer's customers use advance sales discount program.

The objective is to minimize the annual total cost incurred at the retailer,

$\mathrm{TC}(T)=$ Annual ordering cost

+ Annual stock holding cost

+ Annual interest payable-Annualinterestearned

1. Annual ordering cost $=A / T$

2. Annual holding cost $=\frac{\left(1-Y_{1}\right) D_{1} T H}{2}$

3. Annual Interest earned by the retailer 


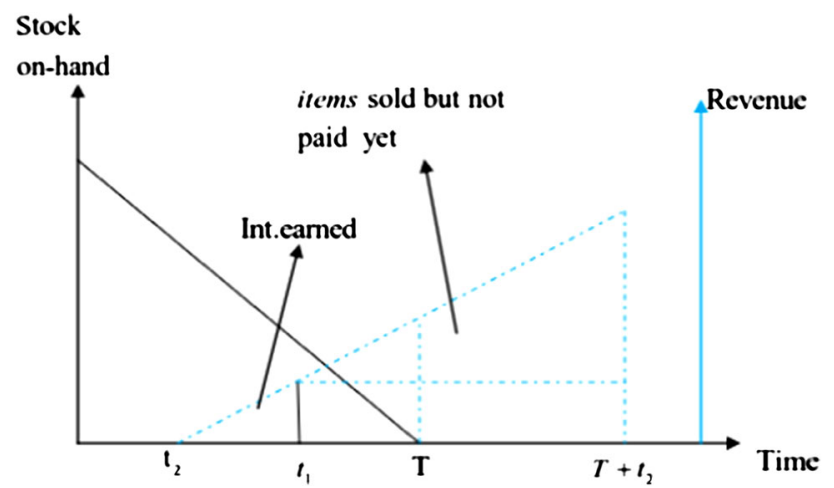

Fig. 1 Interest earned and interest payable for the case $t_{1} \leq T$

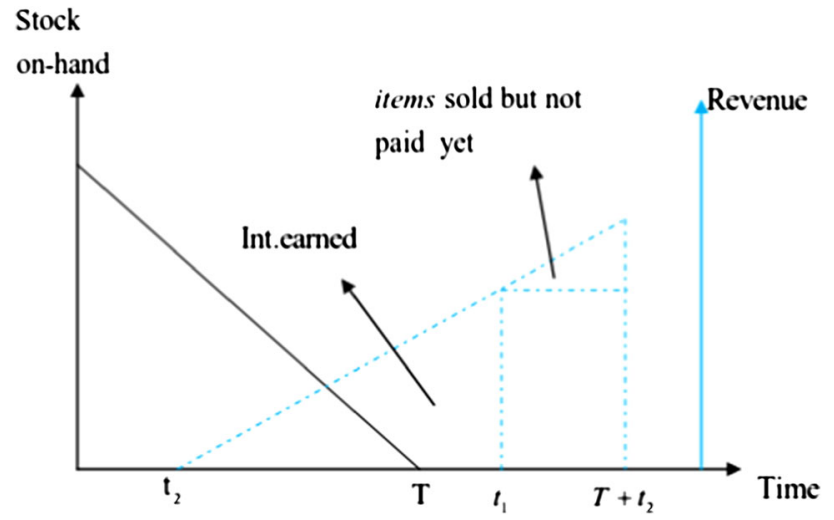

Fig. 2 Interest earned and interest payable for the case $T \leq t_{1} \leq T+t_{2}$

Case 1. When $t_{1} \leq T$ (see Fig. 1$)$. Since $(1-\delta)\left(Y_{1} D_{1}+\right.$ $\left.Y_{2} D_{2}\right)$ number of orders are stand on, the interest earned during the period from $t_{2}$ to $t_{1}$ due to advance sales discount (ASD) program is $(1-\delta)\left(Y_{1} D_{1}+Y_{2} D_{2}\right) p$ $(1-r) I_{\mathrm{e}}\left(t_{1}-t_{2}\right) T$. The interest earned during the normal sales period is.

$p I_{\mathrm{e}}\left(1-Y_{1}\right) D_{1}\left[\frac{\left(t_{1}-t_{2}\right)^{2}}{2}\right]$

The annual interest earned is $(1-\delta)\left(Y_{1} D_{1}+Y_{2} D_{2}\right) p(1$

$$
-r) I_{\mathrm{e}}\left(t_{1}-t_{2}\right)+\frac{p I_{\mathrm{e}}\left(1-Y_{1}\right) D_{1}}{2 T}\left(t_{1}-t_{2}\right)^{2}
$$

Case 2. When $T \leq t_{1} \leq T+t_{2}$ (see Fig. 2). Interest earned due to ASD program is $(1-\delta)\left(Y_{1} D_{1}+Y_{2} D_{2}\right) p(1-r)$ $I_{\mathrm{e}}\left(t_{1}-t_{2}\right) T$. The interest earned during the normal sales period is

$p I_{\mathrm{e}}\left(1-Y_{1}\right) D_{1}\left[\frac{\left(t_{1}-t_{2}\right)^{2}}{2}\right]$.

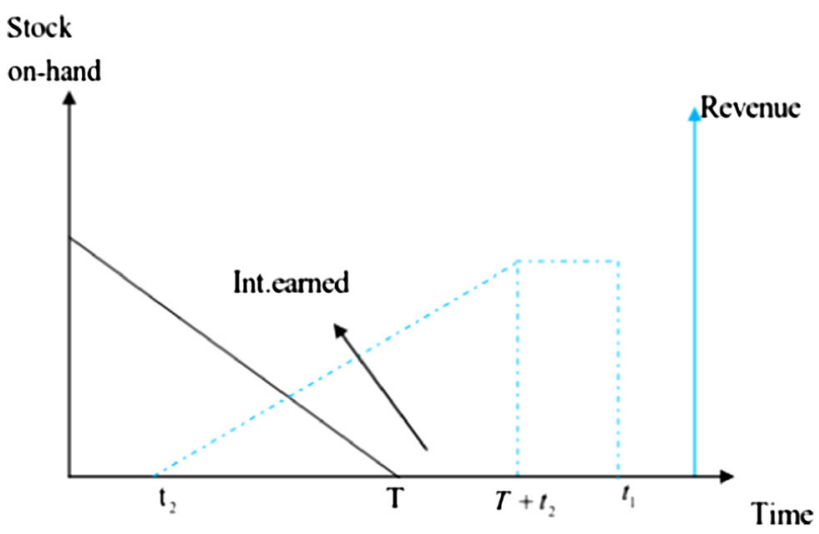

Fig. 3 Interest earned and interest payable for the case $T+t_{2} \leq t_{1}$

The annual interest earned is $(1-\delta)\left(Y_{1} D_{1}+Y_{2} D_{2}\right) p(1$

$$
-r) I_{\mathrm{e}}\left(t_{1}-t_{2}\right)+\frac{p I_{\mathrm{e}}\left(1-Y_{1}\right) D_{1}}{2 T}\left(t_{1}-t_{2}\right)^{2}
$$

Case 3. When $T+t_{2} \leq t_{1}$ (Fig. 3)Interest earned due to ASD program is $(1-\delta)\left(Y_{1} D_{1}+Y_{2} D_{2}\right) p(1-r)$ $I_{\mathrm{e}}\left(t_{1}-t_{2}\right) T$. The interest earned during the normal sales period is

$p I_{\mathrm{e}}\left(1-Y_{1}\right) D_{1}\left[\frac{T^{2}}{2}+T\left(t_{1}-T-t_{2}\right)\right]$

The annual interest earned by the retailer is

$$
\begin{aligned}
& (1-\delta)\left(Y_{1} D_{1}+Y_{2} D_{2}\right) p(1-r) I_{\mathrm{e}}\left(t_{1}-t_{2}\right) \\
& \quad+p I_{\mathrm{e}}\left(1-Y_{1}\right) D_{1}\left[t_{1}-t_{2}-\frac{T}{2}\right]
\end{aligned}
$$

4. Annual Interest payable by the retailer

Case 1. When $t_{1} \leq T$ (see Fig. 1). The interest payable for the items in stock is $c I_{\mathrm{p}}\left(1-Y_{1}\right) D_{1}\left[\frac{\left(T-t_{1}\right)^{2}}{2}\right]$. The interest payable for the items, which are sold but not paid yet, is

$p I_{\mathrm{p}}\left(1-Y_{1}\right) D_{1} t_{2}\left[T-t_{1}+\frac{t_{2}}{2}\right]$.

Therefore, the annual interest payable is

$\frac{c I_{\mathrm{p}}\left(1-Y_{1}\right) D_{1}}{2 T}\left(T-t_{1}\right)^{2}+\frac{p I_{\mathrm{p}}\left(1-Y_{1}\right) D_{1} t_{2}}{T}\left[T-t_{1}+\frac{t_{2}}{2}\right]$

Case 2. When $T \leq t_{1} \leq T+t_{2}$ (see Fig. 2). Since there is no stock on hand, retailer does not need to pay interest for the items in stock. However, he pays interest for the items which are sold, but not paid yet.Therefore, the annual interest payable is 
$\frac{p I_{\mathrm{p}}\left(1-Y_{1}\right) D_{1}}{2 T}\left[T+t_{2}-t_{1}\right]^{2}$

Case 3. When $T+t_{2} \leq t_{1}$. Since the retailer pays off his items at time $t_{1}$, which is later than the time $T+t_{2}$ at which he receives all payment from his customers, there is no interest payable by the retailer. Therefore, the total cost TC $(T)$ incurred at the retailer is

$\mathrm{TC}(T)=\left\{\begin{array}{llc}\mathrm{TC}_{1}(T) & \text { if } & t_{1} \leq T \\ \mathrm{TC}_{2}(T) & \text { if } & T \leq t_{1} \leq T+t_{2} \\ \mathrm{TC}_{3}(T) & \text { if } & T+t_{2} \leq t_{1}\end{array}\right.$

where

$$
\begin{aligned}
\mathrm{TC}_{1}(T)= & \frac{1}{2 T}\left[2 A+\left(1-Y_{1}\right) D_{1}\right. \\
& \left.\times\left[c I_{\mathrm{p}} t_{1}^{2}-p I_{\mathrm{e}}\left(t_{1}-t_{2}\right)^{2}-p I_{\mathrm{p}} t_{2}\left(2 t_{1}-t_{2}\right)\right]\right] \\
& +\frac{T}{2}\left[\left(1-Y_{1}\right) D_{1}\left(c I_{\mathrm{p}}+H\right)\right] \\
& +\left(1-Y_{1}\right) D_{1}\left[p I_{\mathrm{p}} t_{2}-c I_{\mathrm{p}} t_{1}\right] \\
& -(1-\delta)\left(Y_{1} D_{1}+Y_{2} D_{2}\right) P(1-r) I_{\mathrm{e}}\left(t_{1}-t_{2}\right)
\end{aligned}
$$

$$
\begin{aligned}
\mathrm{TC}_{2}(T) & =\frac{1}{2 T}\left[2 A-\left(1-Y_{1}\right) D_{1} p\left(I_{\mathrm{e}}-I_{\mathrm{p}}\right)\left(t_{1}-t_{2}\right)^{2}\right] \\
& +\frac{T}{2}\left[\left(1-Y_{1}\right) D_{1}\left(H+p I_{\mathrm{p}}\right)\right]-p I_{\mathrm{p}}\left(1-Y_{1}\right) \\
& \times D_{1}\left[t_{1}-t_{2}\right]-(1-\delta)\left(Y_{1} D_{1}+Y_{2} D_{2}\right) P(1-r) I_{\mathrm{e}}\left(t_{1}-t_{2}\right)
\end{aligned}
$$

$$
\begin{aligned}
\mathrm{TC}_{3}(T) & =\frac{A}{T}+\frac{T}{2}\left[\left(1-Y_{1}\right) D_{1}\left(H+p I_{\mathrm{e}}\right)\right]-p I_{\mathrm{e}}\left(1-Y_{1}\right) D_{1}\left[t_{1}-t_{2}\right] \\
& -(1-\delta)\left(Y_{1} D_{1}+Y_{2} D_{2}\right) P(1-r) I_{\mathrm{e}}\left(t_{1}-t_{2}\right)
\end{aligned}
$$

\section{Optimal solutions}

When retail price discount rate $r$ is fixed

The first order and second order derivatives of $T C_{i}(T)$, $i=1,2,3$ are as follows:

$$
\begin{aligned}
& \frac{\mathrm{dTC}_{1}(T)}{\mathrm{d} T}=\frac{-1}{2 T^{2}}\left[2 A+\left(1-Y_{1}\right) D_{1}\right. \\
& \left.\left(c I_{\mathrm{p}} t_{1}^{2}-p I_{\mathrm{e}}\left(t_{1}-t_{2}\right)^{2}-p I_{\mathrm{p}} t_{2}\left(2 t_{1}-t_{2}\right)\right)\right] \\
& +\frac{1}{2}\left[\left(1-Y_{1}\right) D_{1}\left(H+c I_{\mathrm{p}}\right)\right]
\end{aligned}
$$

$$
\begin{aligned}
& \frac{\mathrm{d}^{2} \mathrm{TC}_{1}(T)}{\mathrm{d} T^{2}}= \frac{1}{T^{3}}\left[2 A+\left(1-Y_{1}\right) D_{1}\right. \\
&\left.\times\left(c I_{\mathrm{p}} t_{1}^{2}-p I_{\mathrm{e}}\left(t_{1}-t_{2}\right)^{2}-p I_{\mathrm{p}} t_{2}\left(2 t_{1}-t_{2}\right)\right)\right] \\
& \frac{\mathrm{dTC}_{2}(T)}{\mathrm{d} T}= \frac{-1}{2 T^{2}}\left[2 A-p\left(1-Y_{1}\right) D_{1}\left(t_{1}-t_{2}\right)^{2}\left(I_{\mathrm{e}}-I_{\mathrm{p}}\right)\right] \\
&+\frac{1}{2}\left[\left(1-Y_{1}\right) D_{1}\left(H+p I_{\mathrm{p}}\right)\right] \\
& \frac{\mathrm{d}^{2} \mathrm{TC}_{2}(T)}{\mathrm{d} T^{2}}= \frac{1}{T^{3}}\left[2 A-p\left(I_{\mathrm{e}}-I_{\mathrm{p}}\right)\left(1-Y_{1}\right) D_{1}\left(t_{1}-t_{2}\right)^{2}\right] \\
& \frac{\mathrm{dTC}_{3}(T)}{\mathrm{d} T}= \frac{-A}{T^{2}}+\frac{1}{2}\left[\left(1-Y_{1}\right) D_{1}\left(H+p I_{\mathrm{e}}\right)\right] \\
& \frac{\mathrm{d}^{2} \mathrm{TC}_{3}(T)}{\mathrm{d} T^{2}}=\frac{2 A}{T^{3}}>0
\end{aligned}
$$

From the above, we observe that $\mathrm{TC}_{1}(T)$ is a convex function on $T$ if $\left[2 A+\left(1-Y_{1}\right) D_{1}\left(c I_{\mathrm{p}} t_{1}^{2}-p I_{\mathrm{e}}\left(t_{1}-t_{2}\right)^{2}\right.\right.$ $\left.\left.-p I_{\mathrm{p}} t_{2}\left(2 t_{1}-t_{2}\right)\right)\right]>0$. If $\left[2 A+\left(1-Y_{1}\right) D_{1}\left(c I_{\mathrm{p}} t_{1}^{2}-p I_{\mathrm{e}}\left(t_{1}\right.\right.\right.$ $\left.\left.\left.-t_{2}\right)^{2}-p I_{\mathrm{p}} t_{2}\left(2 t_{1}-t_{2}\right)\right)\right]<0$, then $\mathrm{TC}_{1}(T)$ is a concave function on $T$ and $\frac{\mathrm{dTC}_{1}(T)}{\mathrm{d} T}$ is an increasing function on $\left[t_{1}, \infty\right)$. Therefore, minimum $\mathrm{TC}_{1}(T)$ is attained at $T_{1}^{*}=t_{1}$ when $\mathrm{TC}_{1}(T)$ is a concave function of $T . \mathrm{TC}_{2}(T)$ is a convex function on $T$ if $\left[2 A-p\left(I_{\mathrm{e}}-I_{\mathrm{p}}\right)\left(1-Y_{1}\right)\right.$ $\left.D_{1}\left(t_{1}-t_{2}\right)^{2}\right]>0 . \quad$ If $\quad\left[2 A-p\left(I_{\mathrm{e}}-I_{\mathrm{p}}\right)\left(1-Y_{1}\right) D_{1}\right.$ $\left.\left(t_{1}-t_{2}\right)^{2}\right]<0$, then $\mathrm{TC}_{2}(T)$ is a concave function on $T$ and $\frac{\mathrm{dTC}_{2}(T)}{\mathrm{d} T}$ is an increasing function on $\left[t_{1}-t_{2}, t_{1}\right]$. Therefore, minimum $\mathrm{TC}_{2}(T)$ is attained at $T_{2}^{*}=t_{1}-t_{2}$ when $\mathrm{TC}_{2}(T)$ is a concave function of $T$. The optimal cycle times $T_{i}^{*}$ $(i=1,2,3)$ are obtained by solving $\frac{d T C_{i}(T)}{d T}=0(i=1,2$, $3)$, respectively.

$T_{1}^{*}=\left[\frac{2 A+\left(1-Y_{1}\right) D_{1}\left(c I_{\mathrm{p}} t_{1}^{2}-p I_{\mathrm{e}}\left(t_{1}-t_{2}\right)^{2}-p I_{\mathrm{p}} t_{2}\left(2 t_{1}-t_{2}\right)\right)}{\left(1-Y_{1}\right) D_{1}\left(H+c I_{\mathrm{p}}\right)}\right]^{\frac{1}{2}}$

$T_{2}^{*}=\left[\frac{2 A-p\left(1-Y_{1}\right) D_{1}\left(t_{1}-t_{2}\right)^{2}\left(I_{\mathrm{e}}-I_{\mathrm{p}}\right)}{\left(1-Y_{1}\right) D_{1}\left(H+p I_{\mathrm{p}}\right)}\right]^{\frac{1}{2}}$

$T_{3}^{*}=\left[\frac{2 A}{\left(1-Y_{1}\right) D_{1}\left(H+p I_{\mathrm{e}}\right)}\right]^{\frac{1}{2}}$.

Ensuring the condition that $T_{1}^{*} \geq t_{1}$, we have $2 A \geq(1-$ $\left.Y_{1}\right) D_{1}\left(H t_{1}^{2}+p I_{e}\left(t_{1}-t_{2}\right)^{2}+p I_{p} t_{2}\left(2 t_{1}-t_{2}\right)\right)$ if and only if 
$T^{*}=T_{1}^{*}$. Ensuring the condition that $T_{2}^{*} \leq t_{1} \leq T_{2}^{*}+t_{2}$, we have $T^{*}=T_{2}^{*}$ if and only if $2 A \geq\left(1-Y_{1}\right) D_{1}\left(t_{1}-t_{2}\right)^{2}\left(H+p I_{\mathrm{e}}\right)$ and

$$
2 A \leq\left(1-Y_{1}\right) D_{1}\left(H t_{1}^{2}+p I_{\mathrm{e}}\left(t_{1}-t_{2}\right)^{2}+p I_{\mathrm{p}} t_{2}\left(2 t_{1}\right.\right.
$$

$\left.-t_{2}\right)$ ). Ensuring the condition that $T_{3}^{*}+t_{2} \leq t_{1}$, we have $T^{*}=$ $T_{3}^{*}$ if and only if $2 A \leq\left(1-Y_{1}\right) D_{1}\left(t_{1}-t_{2}\right)^{2}\left(H+p I_{\mathrm{e}}\right)$. Let

$$
\begin{array}{r}
\Delta_{1}=\left(1-Y_{1}\right) D_{1}\left(H t_{1}^{2}+p I_{\mathrm{e}}\left(t_{1}-t_{2}\right)^{2}+p I_{\mathrm{p}} t_{2}\left(2 t_{1}-t_{2}\right)\right) \\
\Delta_{2}=\left(1-Y_{1}\right) D_{1}\left(t_{1}-t_{2}\right)^{2}\left(H+p I_{\mathrm{e}}\right)
\end{array} .
$$

It is to observe that $\Delta_{1}-\Delta_{2}=\left(1-Y_{1}\right) D_{1}$ $\left[H\left(t_{1}^{2}-\left(t_{1}-t_{2}\right)^{2}\right)+p I_{\mathrm{p}} t_{2}\left(2 t_{1}-t_{2}\right)\right] \geq 0$ and so $\Delta_{1} \geq \Delta_{2}$.

\section{Theorem 1}

1. If $2 A \geq \Delta_{1}$, then $T^{*}=T_{1}^{*}$

2. If $2 A \leq \Delta_{1}$ and $2 A \geq \Delta_{2}$, then $T^{*}=T_{2}^{*}$

3. If $2 A \leq \Delta_{2}$, then $T^{*}=T_{3}^{*}$

\section{Proof}

(1) $2 A \geq \Delta_{1}$ is implied from $t_{1} \leq T_{1}^{*}$. Since $\mathrm{TC}_{1}^{\prime}(T)$ is an increasing function on $\left[t_{1}, \infty\right), \mathrm{TC}_{1}^{\prime}\left(t_{1}\right) \leq \mathrm{TC}_{1}^{\prime}\left(T_{1}^{*}\right)$. So we have, $\quad \mathrm{TC}_{1}^{\prime}\left(t_{1}\right) \leq 0, \quad \mathrm{TC}_{1}^{\prime}\left(T_{1}^{*}\right)=0 \quad$ and $\mathrm{TC}_{1}^{\prime}(T)>0$ for $T \in\left[T_{1}^{*}, \infty\right)$. Thus,

$\mathrm{TC}_{1}(T)$ is decreasing on $\left[t_{1}, T_{1}^{*}\right]$ and increasing on $\left[T_{1}^{*}, \infty\right)$.

Since $2 A \geq \Delta_{1}$ is implied from $T_{2}^{*} \geq t_{1}$. Since $\mathrm{TC}_{2}^{\prime}(T)$ is an increasing function, $\mathrm{TC}_{2}^{\prime}\left(T_{2}^{*}\right) \geq \mathrm{TC}_{2}^{\prime}\left(t_{1}\right)$ which implies $\mathrm{TC}_{2}^{\prime}\left(t_{1}\right) \leq 0 . \quad t_{1}-t_{2} \leq t_{1}$ implies $\mathrm{TC}_{2}^{\prime}\left(t_{1}-t_{2}\right) \leq \mathrm{TC}_{2}^{\prime}\left(t_{1}\right)$. Hence, $\mathrm{TC}_{2}(T)$ is decreasing on $\left[t_{1}-t_{2}, t_{1}\right]$.

$2 A \geq \Delta_{1}$ implies $2 A \geq \Delta_{2} .2 A \geq \Delta_{2}$ is implied from $T_{3}^{*} \geq t_{1}-t_{2}$. Since $\mathrm{TC}_{3}^{\prime}(T)$ is an increasing function, $\mathrm{TC}_{3}^{\prime}\left(t_{1}-t_{2}\right) \leq \mathrm{TC}_{3}^{\prime}\left(T_{3}^{*}\right)$ which implies $\mathrm{TC}_{3}^{\prime}\left(t_{1}-t_{2}\right) \leq 0$. Since $\mathrm{TC}_{3}^{\prime}(0) \leq 0, \quad \mathrm{TC}_{3}(T)$ is decreasing on $\left[0, t_{1}-t_{2}\right]$. From the discussions, we have

(i) $\mathrm{TC}_{1}(T)$ is decreasing on $\left[t_{1}, T_{1}^{*}\right]$ and increasing on $\left[T_{1}^{*}, \infty\right)$.

(ii) $\quad \mathrm{TC}_{2}(T)$ is decreasing on $\left[t_{1}-t_{2}, t_{1}\right]$

(iii) $\quad \mathrm{TC}_{3}(T)$ is decreasing on $\left[0, t_{1}-t_{2}\right]$.

Therefore, $\mathrm{TC}(T)$ attains minimum at $T^{*}=T_{1}^{*}$ and $T C^{*}(T)=T C_{1}\left(T_{1}^{*}\right)$.

(2). Let $2 A \leq \Delta_{1}$ and $2 A \geq \Delta_{2} \cdot 2 A \leq \Delta_{1}$ is implied from $t_{1} \geq T_{1}^{*}$. So, $\mathrm{TC}_{1}^{\prime}\left(t_{1}\right) \geq 0$. Since $\mathrm{TC}_{1}^{\prime}(T)$ is an increasing function on $\left[t_{1}, \infty\right), \mathrm{TC}_{1}^{\prime}(T) \geq 0$ for $T \in\left[t_{1}, \infty\right)$. Therefore, $\mathrm{TC}_{1}(T)$ is increasing on $\left[t_{1}, \infty\right) .2 A \leq \Delta_{1}$ can also be implied from $t_{1} \geq T_{2}^{*}$. $2 A \geq \Delta_{2}$ is implied from $t_{1}-t_{2} \leq T_{2}^{*}$. Since $\mathrm{TC}_{2}^{\prime}(T)$ is an increasing function, $\mathrm{TC}_{2}^{\prime}\left(t_{1}\right) \geq$
$\mathrm{TC}_{2}^{\prime}\left(T_{2}^{*}\right) \quad$ and $\quad \mathrm{TC}_{2}^{\prime}\left(t_{1}-t_{2}\right) \leq \mathrm{TC}_{2}^{\prime}\left(T_{2}^{*}\right)$. So, $\mathrm{TC}_{2}^{\prime}\left(t_{1}\right) \geq 0$ and $T C_{2}^{\prime}\left(t_{1}-t_{2}\right) \leq 0$; Since $T C_{2}^{\prime}\left(T_{2}^{*}\right)$ $=0, T C_{2}(T)$ is decreasing on $\left[t_{1}-t_{2}, T_{2}^{*}\right]$ and increasing on $\left[T_{2}^{*}, t_{1}\right] .2 A \geq \Delta_{2}$ can be implied from $T_{3}^{*} \geq t_{1}-t_{2}$. Since $\mathrm{TC}_{3}^{\prime}(T)$ is an increasing function $\mathrm{TC}_{3}^{\prime}\left(T_{3}^{*}\right) \geq \mathrm{TC}_{3}^{\prime}\left(t_{1}-t_{2}\right)$ which implies $\mathrm{TC}_{3}^{\prime}\left(t_{1}-\right.$ $\left.t_{2}\right) \leq 0$. Since $\mathrm{TC}_{3}^{\prime}(T)$ is increasing on $\left[0, t_{1}-t_{2}\right]$, $\mathrm{TC}_{3}^{\prime}(T) \leq 0$ for $T \in\left[0, t_{1}-t_{2}\right]$. Therefore, $\mathrm{TC}_{3}(T)$ is decreasing on $T \in\left[0, t_{1}-t_{2}\right]$. Hence, we have

(i) $\quad \mathrm{TC}_{1}(T)$ is decreasing on $\left[t_{1}, \infty\right)$

(ii) $\mathrm{TC}_{2}(T)$ is decreasing on $\left[t_{1}-t_{2}, T_{2}^{*}\right]$ and increasing on $\left[T_{2}^{*}, t_{1}\right]$.

(iii) $\quad \mathrm{TC}_{3}(T)$ is decreasing on $\left[0, t_{1}-t_{2}\right]$.

Therefore, $\operatorname{TC}(T)$ attains minimum at $T^{*}=T_{2}^{*}$ and $\mathrm{TC}^{*}(T)=\mathrm{TC}_{2}\left(T_{2}^{*}\right)$.

(3) $2 A \leq \Delta_{2}$ implies $2 A \leq \Delta_{1} .2 A \leq \Delta_{1}$ implies that $\mathrm{TC}_{1}(T)$ is increasing on $\left[t_{1}, \infty\right) .2 A \leq \Delta_{2}$ can be implied from $t_{1}-t_{2} \geq T_{2}^{*}$. Since $\mathrm{TC}_{2}^{\prime}(T)$ is increasing, $\quad \mathrm{TC}_{2}^{\prime}\left(t_{1}-t_{2}\right) \geq \mathrm{TC}_{2}^{\prime}\left(T_{2}^{*}\right)$. Therefore, $\mathrm{TC}_{2}^{\prime}\left(t_{1}-t_{2}\right) \geq 0 . \quad 2 A \leq \Delta_{1} \quad$ is implied from $t_{1} \geq T_{2}^{*}$. Thus, $\quad \mathrm{TC}_{2}^{\prime}\left(t_{1}\right) \geq \mathrm{TC}_{2}^{\prime}\left(T_{2}^{*}\right)$. Therefore, $\mathrm{TC}_{2}^{\prime}\left(t_{1}\right) \geq 0$. Therefore, $\mathrm{TC}_{2}(T)$ is increasing on $\left[t_{1}-t_{2}, t_{1}\right] .2 A \leq \Delta_{2}$ implies $\mathrm{TC}_{3}(T)$ is a convex function on $\left[0, t_{1}-t_{2}\right]$. Therefore, $\mathrm{TC}_{3}(T)$ is decreasing on $\left[0, T_{3}^{*}\right]$ and increasing on $\left[T_{3}^{*}, t_{1}-t_{2}\right]$. From the above discussion, we have

(i) $\mathrm{TC}_{1}(T)$ is increasing on $\left[t_{1}, \infty\right)$

(ii) $\mathrm{TC}_{2}(T)$ is increasing on $\left[t_{1}-t_{2}, t_{1}\right]$

(iii) $\quad \mathrm{TC}_{3}(T)$ is decreasing on $\left[0, T_{3}^{*}\right]$ and increasing on $\left[T_{3}^{*}, t_{1}-t_{2}\right]$.

Hence, $\operatorname{TC}(T)$ attains minimum at $T^{*}=T_{3}^{*}$ and $\mathrm{TC}^{*}(T)=\mathrm{TC}_{3}\left(T_{3}^{*}\right)$.

When retail price discount rate is endogenous

Here, the retailer determines the optimal replenishment cycle time $T^{*}$ and the optimal price discount $r^{*}$ to minimize TC $(T)$. With the consideration that the retailer 1 's demand due to advance sales discount program and the fraction of other retailers' customers who switch to retailer 1 under advance sales discount program are linearly increasing with retail price discount $r$, let $Y_{1}(r)=\alpha \cdot r$ and $Y_{2}(r)=$ $\beta \cdot r$ as in Tsao (2009). The problem, here, is to minimize

$\mathrm{TC}(T, r)=\left\{\begin{array}{llr}\mathrm{TC}_{1}(T, r) & \text { if } & t_{1} \leq T \\ \mathrm{TC}_{2}(T, r) & \text { if } & T \leq t_{1} \leq T+t_{2} \\ \mathrm{TC}_{3}(T, r) & \text { if } & T+t_{2} \leq t_{1}\end{array}\right.$

To solve this problem, the closed-form solution for each $r_{i}(T)$, is found by solving $\frac{\partial \operatorname{TTC}_{i}(T, r)}{\partial r}=0, i=1,2,3$. Substituting these $r_{i}(T)$ to the corresponding $T C_{i}(T, r), \mathrm{TC}_{i}(T, r)$ 
reduces to single variable function as $\mathrm{TC}_{i}(T)$. The optimal value of $T_{i}^{*}$ is determined by solving the $\frac{\operatorname{dTC}_{i}\left(T, r_{i}(T)\right)}{\partial T}=0$, $i=1,2,3$. The optimal values of $T^{*}$ and $r^{*}$ are such that $\mathrm{TC}\left(T^{*}, r^{*}\right)=\min \left\{\mathrm{TC}_{1}\left(T_{1}^{*}, r_{1}^{*}\right), \mathrm{TC}_{2}\left(T_{2}^{*}, r_{2}^{*}\right), \mathrm{TC}_{3}\left(T_{3}^{*}, r_{3}^{*}\right)\right\}$.

The second derivative of $\mathrm{TC}_{i}(T, r)$ with respect to $\mathrm{r}$ is $\frac{\partial^{2} T C_{i}(T, r)}{\partial r^{2}}=2(1-\delta)\left(\alpha D_{1}+\beta D_{2}\right) p I_{e}\left(t_{1}-t_{2}\right)>0 . \quad$ Thus, $T C_{i}(T, r),(i=1,2,3)$ is a convex function of $\mathrm{r}$ for a fixed value of $T$. Solving the Partial differential equation $\frac{\partial \mathrm{TC}_{i}(T, r)}{\partial r}=0, i=1,2,3$, we get

$$
\begin{aligned}
r_{1}(T)= & \frac{1}{4(1-\delta)\left(\alpha D_{1}+\beta D_{2}\right) p I_{\mathrm{e}}\left(t_{1}-t_{2}\right)} \\
& \times\left[\begin{array}{c}
2(1-\delta)\left(\alpha D_{1}+\beta D_{2}\right) p I_{\mathrm{e}}\left(t_{1}-t_{2}\right) \\
+\frac{\alpha D_{1}}{T}\left(\begin{array}{c}
c I_{\mathrm{p}} t_{1}^{2}-p I_{\mathrm{e}}\left(t_{1}-t_{2}\right)^{2} \\
-p I_{\mathrm{p}} t_{2}\left(2 t_{1}-t_{2}\right)
\end{array}\right) \\
+\alpha D_{1} T\left(H+c I_{\mathrm{p}}\right)+2 \alpha D_{1}\left(p I_{\mathrm{p}} t_{2}-c I_{\mathrm{p}} t_{1}\right)
\end{array}\right]
\end{aligned}
$$

$$
\begin{aligned}
r_{2}(T)= & \frac{1}{4(1-\delta)\left(\alpha D_{1}+\beta D_{2}\right) p I_{\mathrm{e}}\left(t_{1}-t_{2}\right)} \\
& \times\left[\begin{array}{c}
2(1-\delta)\left(\alpha D_{1}+\beta D_{2}\right) p I_{\mathrm{e}}\left(t_{1}-t_{2}\right) \\
+\frac{\alpha D_{1}}{T}\left(\left(I_{\mathrm{e}}+I_{\mathrm{p}}\right) p\left(t_{1}-t_{2}\right)^{2}\right) \\
+\alpha D_{1} T\left(H+c I_{\mathrm{p}}\right)-2 \alpha D_{1} p I_{\mathrm{p}}\left(t_{1}-t_{2}\right)
\end{array}\right]
\end{aligned}
$$

$$
\begin{aligned}
& r_{3}(T)=\frac{1}{4(1-\delta)\left(\alpha D_{1}+\beta D_{2}\right) p I_{\mathrm{e}}\left(t_{1}-t_{2}\right)} \\
& {\left[\alpha D_{1} T H-2 \alpha D_{1} p I_{\mathrm{e}}\left(t_{1}-t_{2}-T / 2\right)+2(1-\delta)\left(\alpha D_{1}\right.\right.} \\
& \left.\left.+\beta D_{2}\right) p I_{\mathrm{e}}\left(t_{1}-t_{2}\right)\right]
\end{aligned}
$$

\section{Theorem 2}

(a) If $-\frac{\alpha D_{1}}{2 T}\left(c I_{\mathrm{p}} t_{1}^{2}-P I_{\mathrm{e}}\left(t_{1}-t_{2}\right)^{2}-p I_{\mathrm{p}} t_{2}\left(2 t_{1}-t_{2}\right)\right)$ $-\frac{\alpha D_{1}}{2} T\left(H+c I_{\mathrm{p}}\right)-\alpha D_{1}\left(P I_{\mathrm{p}} t_{2}-c I_{\mathrm{p}} t_{1}\right)+(1-\delta)$ $\left(\alpha D_{1}+\beta D_{2}\right) P I_{\mathrm{e}}\left(t_{1}-t_{2}\right)>0$ then unique optimal unique optimal solution $r_{1}^{*}(T)$ lies in the interval $(0,1)$.

(b) If $-\frac{\alpha D_{1}}{2 T}\left(\left(I_{\mathrm{e}}+I_{\mathrm{p}}\right) p\left(t_{1}-t_{2}\right)^{2}\right)-\frac{\alpha D_{1}}{2} T\left(H+p I_{\mathrm{p}}\right)$ $+\alpha D_{1} p I_{\mathrm{p}}\left(t_{1}-t_{2}\right)+(1-\delta)\left(\alpha D_{1}+\beta D_{2}\right) p I_{\mathrm{e}}\left(t_{1}-t_{2}\right)$ $>0$ then unique optimal solution $r_{2}^{*}(T)$ lies in the interval $(0,1)$.

(c) If $-\frac{\alpha D_{1}}{2 T}\left(\left(I_{\mathrm{e}}+I_{\mathrm{p}}\right) p\left(t_{1}-t_{2}\right)^{2}\right)-\frac{\alpha D_{1}}{2} T\left(H+p I_{\mathrm{p}}\right)$ $+\alpha D_{1} p I_{\mathrm{p}}\left(t_{1}-t_{2}\right)+(1-\delta)\left(\alpha D_{1}+\beta D_{2}\right) p I_{\mathrm{e}}\left(t_{1}-t_{2}\right)$ $>0$ then unique optimal solution $r_{3}^{*}(T)$ lies in the interval $(0,1)$.

\section{Proof}

(a) Let

$$
\begin{aligned}
G_{1}(r)= & \frac{\partial \mathrm{TC}_{1}(T, r)}{\partial r} \\
= & -\frac{\alpha D_{1}}{2 T}\left(c I_{\mathrm{p}} t_{1}^{2}-p I_{\mathrm{e}}\left(t_{1}-t_{2}\right)^{2}-p I_{\mathrm{p}} t_{2}\left(2 t_{1}-t_{2}\right)\right) \\
& -\frac{\alpha D_{1}}{2} T\left(H+c I_{\mathrm{p}}\right)-\alpha D_{1}\left(p I_{\mathrm{p}} t_{2}-c I_{\mathrm{p}} t_{1}\right) \\
& -(1-\delta)\left(\alpha D_{1}+\beta D_{2}\right) p(1-2 r) I_{\mathrm{e}}\left(t_{1}-t_{2}\right)
\end{aligned}
$$

Since $\mathrm{TC}_{1}(T, r)$ is a convex function of $r, G_{1}(r)$ is an increasing function of $r$.

$$
\begin{aligned}
G_{1}(0)= & -\frac{\alpha D_{1}}{2 T}\left(c I_{\mathrm{p}} t_{1}^{2}-p I_{\mathrm{e}}\left(t_{1}-t_{2}\right)^{2}-p I_{\mathrm{p}} t_{2}\left(2 t_{1}-t_{2}\right)\right) \\
& -\frac{\alpha D_{1}}{2} T\left(H+c I_{\mathrm{p}}\right)-\alpha D_{1}\left(p I_{\mathrm{p}} t_{2}-c I_{\mathrm{p}} t_{1}\right) \\
& -(1-\delta)\left(\alpha D_{1}+\beta D_{2}\right) p I_{\mathrm{e}}\left(t_{1}-t_{2}\right)<0 \\
G_{1}(1)= & -\frac{\alpha D_{1}}{2 T}\left(c I_{\mathrm{p}} t_{1}^{2}-P I_{\mathrm{e}}\left(t_{1}-t_{2}\right)^{2}-p I_{\mathrm{p}} t_{2}\left(2 t_{1}-t_{2}\right)\right) \\
& -\frac{\alpha D_{1}}{2} T\left(H+c I_{\mathrm{p}}\right)-\alpha D_{1}\left(P I_{\mathrm{p}} t_{2}-c I_{\mathrm{p}} t_{1}\right) \\
& +(1-\delta)\left(\alpha D_{1}+\beta D_{2}\right) P I_{\mathrm{e}}\left(t_{1}-t_{2}\right)
\end{aligned}
$$

If $G_{1}(1)>0$, then $r_{1}^{*}(T)$ lies in the interval $(0,1)$.

(b) Let

$$
\begin{aligned}
G_{2}(r)= & \frac{\partial \mathrm{TC}_{2}(T, r)}{\partial r}=-\frac{\alpha D_{1}}{2 T}\left(\left(I_{\mathrm{e}}+I_{\mathrm{p}}\right) p\left(t_{1}-t_{2}\right)^{2}\right) \\
& -\frac{\alpha D_{1}}{2} T\left(H+p I_{\mathrm{p}}\right)+\alpha D_{1} p I_{\mathrm{p}}\left(t_{1}-t_{2}\right) \\
& -(1-\delta)\left(\alpha D_{1}+\beta D_{2}\right) p(1-2 r) I_{\mathrm{e}}\left(t_{1}-t_{2}\right)
\end{aligned}
$$

Since $\mathrm{TC}_{2}(T, r)$ is a convex function of $r, G_{2}(r)$ is an increasing function of $r$.

$$
\begin{aligned}
G_{2}(0)= & -\frac{\alpha D_{1}}{2 T}\left(\left(I_{\mathrm{e}}+I_{\mathrm{p}}\right) p\left(t_{1}-t_{2}\right)^{2}\right)-\frac{\alpha D_{1}}{2} T H \\
& -\frac{\alpha D_{1} p I_{\mathrm{p}}}{2}\left(T-2\left(t_{1}-t_{2}\right)\right)+\alpha D_{1} p I_{\mathrm{p}}\left(t_{1}-t_{2}\right) \\
& -(1-\delta)\left(\alpha D_{1}+\beta D_{2}\right) p I_{\mathrm{e}}\left(t_{1}-t_{2}\right)<0 \\
G_{2}(1)= & -\frac{\alpha D_{1}}{2 T}\left(\left(I_{\mathrm{e}}+I_{\mathrm{p}}\right) p\left(t_{1}-t_{2}\right)^{2}\right) \\
& -\frac{\alpha D_{1}}{2} T\left(H+p I_{\mathrm{p}}\right)+\alpha D_{1} p I_{\mathrm{p}}\left(t_{1}-t_{2}\right) \\
& +(1-\delta)\left(\alpha D_{1}+\beta D_{2}\right) p I_{\mathrm{e}}\left(t_{1}-t_{2}\right)
\end{aligned}
$$

If $G_{2}(1)>0$, then $r_{2}^{*}(T)$ lies in the interval $(0,1)$. 
(c) Similar to (b)

First, we find $r_{1}(T), r_{2}(T)$ and $r_{3}(T)$ using the Eqs. (8), (9) and (10), respectively. Substituting $r_{1}(T), r_{2}(T)$ and $r_{3}(T)$ into $\mathrm{TC}_{1}(r, T), \mathrm{TC}_{2}(r, T)$, and $\mathrm{TC}_{3}(r, T)$, respectively, each $\mathrm{TC}_{i}(r, T)$ becomes a function of $T$ alone rather than a function of $r$ and $T$; since $r$ is a function of $T$. Solving the equations $\frac{\mathrm{dTC}_{i}}{\mathrm{~d} T}=0,(i=1,2,3)$, we get the optimal solutions $T_{1}^{*}, T_{2}^{*}$ and $T_{3}^{*}$.

Find the minimum of $\left\{\mathrm{TC}_{1}\left(r_{1}\left(T_{1}^{*}\right), T_{1}^{*}\right), \mathrm{TC}_{2}\left(r_{2}\left(T_{2}^{*}\right)\right.\right.$, $\left.\left.T_{2}^{*}\right), \mathrm{TC}_{3}\left(r_{3}\left(T_{3}^{*}\right), T_{3}^{*}\right)\right\}$ and the resultant is the optimal cost and corresponding $T_{i}^{*}$ and $r_{i}\left(T_{i}^{*}\right)$ are the optimal solutions. While we are solving the differential equation $\frac{\mathrm{dTC}_{i}}{\mathrm{~d} T}=0$, if we get multiple solutions, then we have to check the $\frac{\mathrm{d}^{2} \mathrm{TC}_{i}}{\mathrm{~d} T^{2}}>0$ for optimality

\section{Numerical Analysis}

Here, we find the optimal solutions for various cases to illustrate the solution procedures and obtain sensitivity analysis on order cancelation rate $(\delta)$, ordering cost $(A)$ and holding cost $(H)$, trade credit periods $t_{1}$ and $t_{2}$.

Example 1 Let $A=300, \quad D_{1}=2,000, D_{2}=2,000$, $\delta=0.1, H=1, I_{\mathrm{p}}=0.15, I_{\mathrm{e}}=0.2, t_{1}=0.14, t_{2}=0.10$, $p=11, c=10$. First, let $r$ be fixed, say $r=0.56$. We get $\Delta_{1}=453.80, \Delta_{2}=7.25$. Clearly $2 A>\Delta_{1}$ and by Theorem 1, we obtain that $T_{1}^{*}=0.4096$ and the total cost $\mathrm{TC}_{1}=1,347.60$. If $r$ is a decision variable, then we utilize the solution procedure in Sect. "When retail price discount rate is endogenous". The optimal solutions are $T_{1}^{*}=0.5560, r_{1}^{*}=0.8963$ and $\mathrm{TC}_{1}^{*}=1,214.80$. A graphic representation of $\mathrm{TC}_{i}$ is shown in Fig. 4.

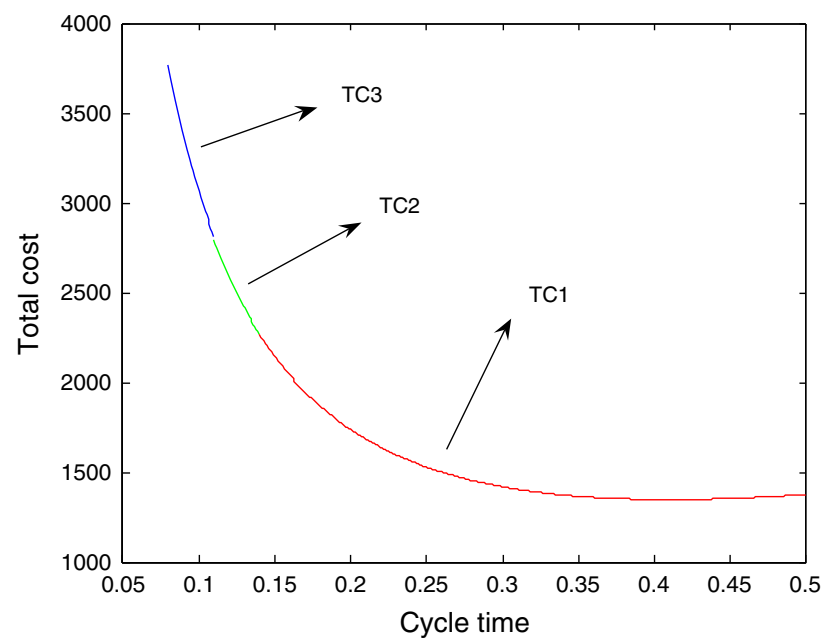

Fig. 4 Graphic representation of $\mathrm{TC}_{i}$ for example 1

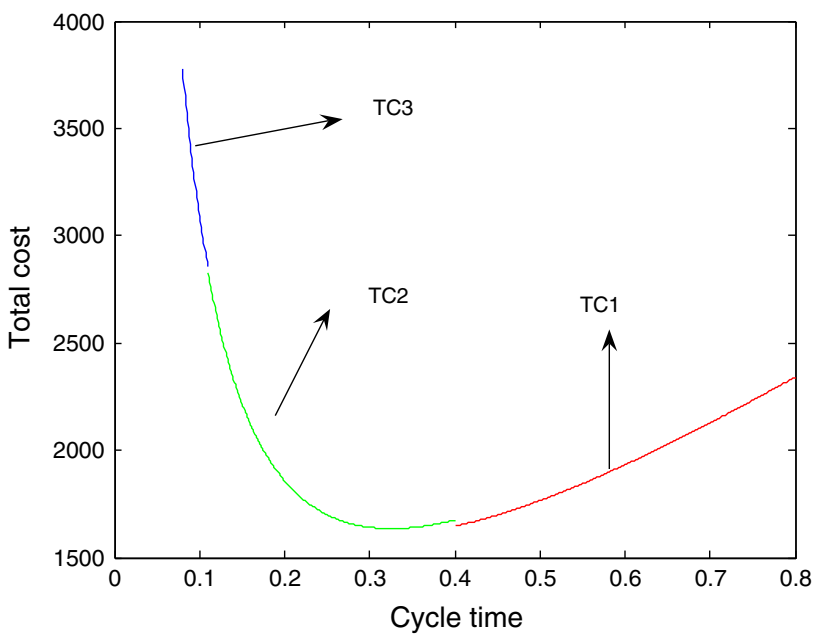

Fig. 5 Graphic representation of $\mathrm{TC}_{i}$ for example 2

Example 2 Let $A=300, \quad D_{1}=3,000, \quad D_{2}=3,000$, $\delta=0.1, H=1, I_{\mathrm{p}}=0.15, I_{\mathrm{e}}=0.2, t_{1}=0.14, t_{2}=0.10$, $p=11, c=10$. First, let $r$ be fixed, say $r=0.56$. We get $\Delta_{1}=680.7, \Delta_{2}=10.9$. Clearly $\Delta_{1}>2 A \& 2 A<\Delta_{2}$ and by Theorem 1 , we obtain that $T_{2}^{*}=0.3354$ and the total cost $\mathrm{TC}_{2}=1,582.50$. If $r$ is a decision variable, then we utilize the solution procedure in Sect. "When retail price discount rate is endogenous". The optimal solutions are $r_{2}^{*}=0.8613, T_{2}^{*}=0.4899$ and $\mathrm{TC}_{1}^{*}=1,486.70$. A graphic representation of $\mathrm{TC}_{\mathrm{i}}$ is shown in Fig. 5.

Example 3 Let $A=300, \quad D_{1}=8,000, \quad D_{2}=8,000$, $\delta=0.1, H=1, I_{\mathrm{p}}=0.15, I_{\mathrm{e}}=0.2, t_{1}=0.28, t_{2}=0.09$, $p=11, c=10$. First, let $r$ be fixed, say $r=0.56$. We get $\Delta_{1}=5,292, \Delta_{2}=655$. Clearly $\Delta_{2}>2 A$ and by Theorem 1, we obtain that $T_{3}^{*}=0.1818$ and the total cost $\mathrm{TC}_{2}=165.53$. If $\mathrm{r}$ is a decision variable, then we utilize the solution procedure in Sect. "When retail price discount rate is endogenous". The optimal solutions are $r_{2}^{*}=0.5611, T_{2}^{*}=0.5573$ and $\mathrm{TC}_{1}^{*}=245.80$. A graphic representation of $\mathrm{TC}_{i}$ is shown in Fig. 6.

\section{Sensitivity analysis}

Here, we consider the data as in numerical Example 2. Sensitivity analysis on various parameters is presented in the Table 1. It is also illustrated in Figs. 7, 8, 9, 10, 11.

Based on the results in Table 1 and Figs. 7, 8, 9, 10, 11 and the following are observed:

- When partial order cancelation rate $\delta$ increases, the cycle time and total cost are decreased, whereas the optimal price discount rate increases marginally to increase the sales marginally.

- When holding cost $H$ increases, the retailer will increase the price discount and shorten the cycle time. 


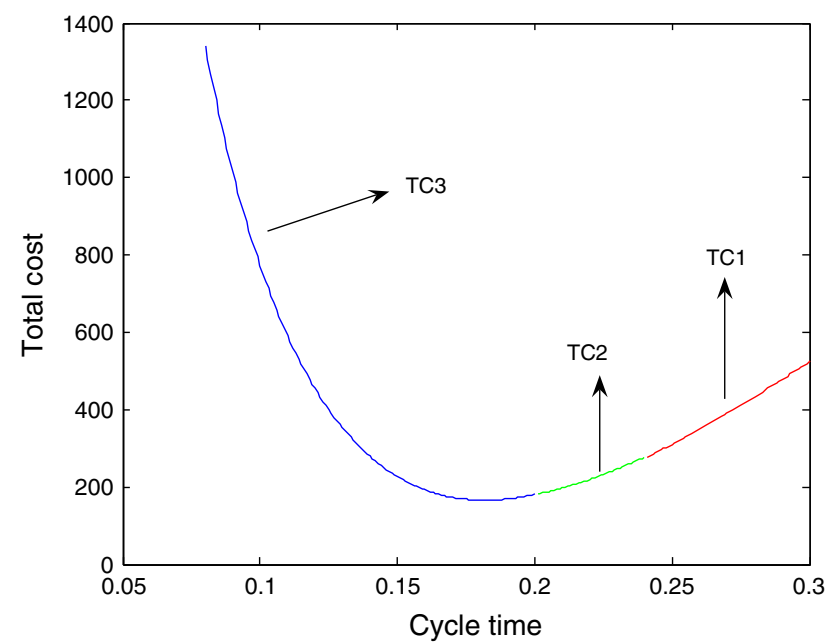

Fig. 6 Graphic representation of $\mathrm{TC}_{i}$ for example 3

Table 1 Sensitivity analysis with respect to the model parameters

\begin{tabular}{lllll}
\hline Parameter & Value & $T^{*}$ & $r^{*}$ & TC $^{*}$ \\
\hline$\delta$ & 0.1 & 0.4899 & 0.8613 & $1,486.70$ \\
& 0.2 & 0.4710 & 0.8893 & $1,457.00$ \\
& 0.25 & 0.4609 & 0.9055 & $1,441.70$ \\
& 0.3 & 0.4504 & 0.9237 & $1,426.00$ \\
& 0.4 & 0.4278 & 0.9671 & $1,393.60$ \\
& 1 & 0.4899 & 0.8613 & $1,486.70$ \\
& 1.2 & 0.4667 & 0.8712 & $1,539.30$ \\
& 1.25 & 0.4613 & 0.8736 & $1,552.10$ \\
& 1.3 & 0.4561 & 0.8760 & $1,564.80$ \\
& 1.4 & 0.4461 & 0.8808 & $1,589.70$ \\
& 300 & 0.4899 & 0.8613 & $1,486.70$ \\
& 310 & 0.4953 & 0.8656 & $1,508.40$ \\
& 315 & 0.4979 & 0.8677 & $1,519.10$ \\
& 320 & 0.5005 & 0.8698 & $1,529.70$ \\
& 330 & 0.5057 & 0.8740 & $1,550.70$ \\
& 0.11 & 0.3359 & 0.8465 & $1,735.60$ \\
& 0.12 & 0.3358 & 0.8465 & $1,684.90$ \\
& 0.13 & 0.3357 & 0.8465 & $1,633.90$ \\
& 0.14 & 0.3354 & 0.8465 & $1,582.50$ \\
& 0.15 & 0.3351 & 0.8465 & $1,530.80$ \\
& 0.05 & 0.3333 & 0.6468 & $1,320.30$ \\
& 0.06 & 0.3339 & 0.6468 & $1,373.50$ \\
& & 0.3343 & 0.6468 & $1,426.30$ \\
& 0.09 & 0.3348 & 0.6468 & $1,478.70$ \\
& & 0.3351 & 0.6468 & $1,530.80$ \\
& & & & \\
& & & &
\end{tabular}

Total cost marginally increases due to the marginal increase in price discount rate offered by the retailer.

- When the ordering cost $A$ increases, the retailer will increase his price discount rate and the inventory cycle

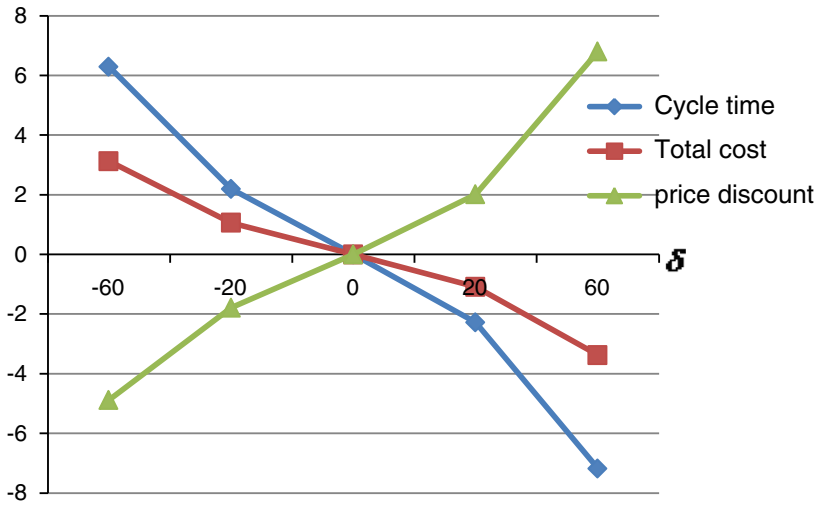

Fig. 7 Effect of percentage (\%) change in $\delta$ versus percentage changes in optimal cycle time, optimal price discount $(r)$, and optimal total cost (TC)

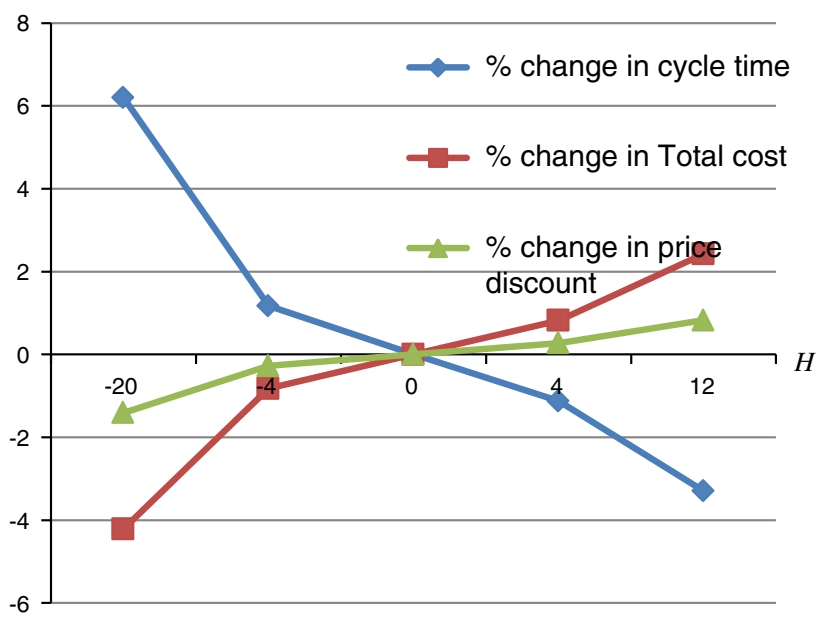

Fig. 8 Effect of $\%$ change in $H$ versus $\%$ changes in optimal cycle time $(T)$, optimal price discount $(r)$, and optimal total cost (TC)

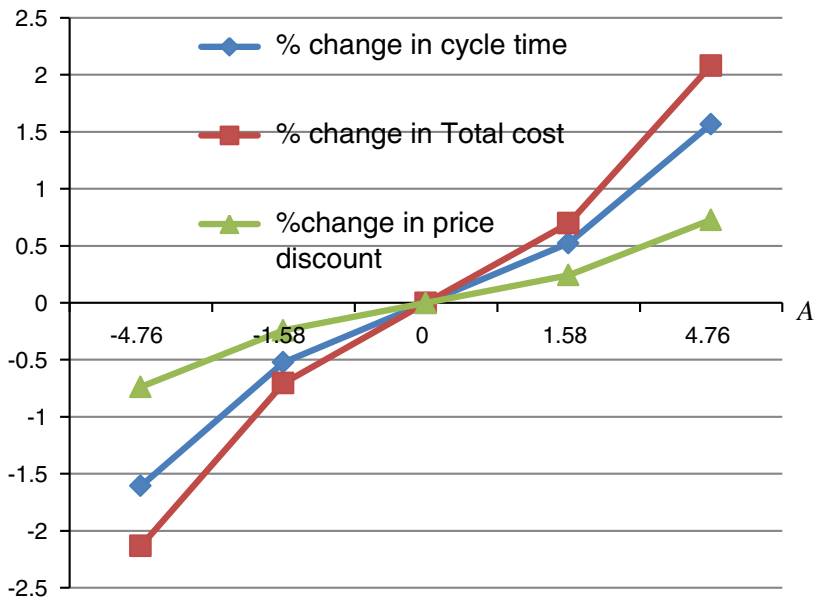

Fig. 9 Effect of $\%$ change in $A$ versus $\%$ changes in optimal cycle time $(T)$, optimal price discount $(r)$, and optimal total cost (TC) 


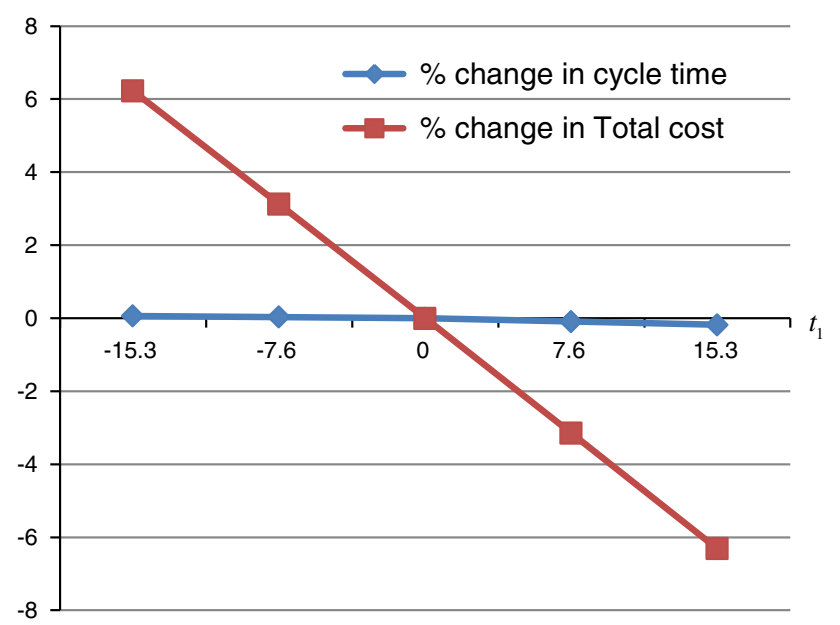

Fig. 10 Effect of $\%$ change in $t_{1}$ versus $\%$ change in optimal cycle time $(T)$, and optimal total cost (TC)

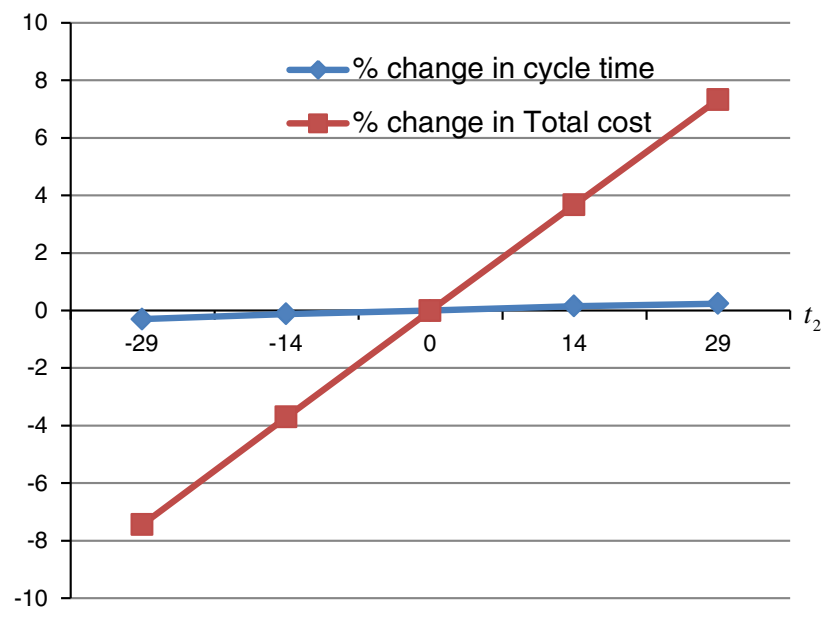

Fig. 11 Effect of $\%$ change in $t_{2}$ versus $\%$ change in optimal cycle time $(T)$, and optimal total cost (TC)

time is also increased. To reduce the frequency of replenishment, the retailer lengthens the cycle time.

- When supplier provides a longer credit period $t_{1}$, the retailer replenishes the goods more often. In other words, retailer will minimize the inventory cycle time to take advantage of longer credit period.

- When retailer provides longer credit period $t_{2}$, the retailer's cycle time will be increased. Thus, the retailer will replenish the goods not often to decrease the loss.

\section{Conclusions and future research}

Unlike, the existing research in two-level trade credit EOQ models, this paper considers partial order cancelation during advance sales period in the retailer's inventory system. The objective behind this consideration is that the market customers' decisions upon their orders play a vital role in trade. Since the payment time of the customers has an impact in the interest earned and interest payable by the retailer, the total costs are estimated under the investigation of exact payment time. Solution procedures are obtained for two cases: (a) when price discount rate is fixed and (b) when price discount rate is a decision variable. Using the derived mathematical theorems, the optimal solutions for price discount and replenishment cycle time are found out for the both cases when price discount rate is fixed and endogenous. The sensitivity analyses are made for various inventory parameters.

In future research, one can implement the effect of inflation, perishability to this paper. Considering the trade credit periods $t_{1}$ and $t_{2}$ as decision variables could be a good extension of this paper. Further, one can improve the paper by considering integrated supplier-retailer inventory system.

Acknowledgments The author thanks two anonymous reviewers for their constructive commentsto improve the paper.

Conflict of interest The author declare that he has no competing interests.

Open Access This article is distributed under the terms of the Creative Commons Attribution License which permits any use, distribution, and reproduction in any medium, provided the original author(s) and the source are credited.

\section{References}

Aggarwal SP, Jaggi CK (1995) Ordering policies of deteriorating items under permissible delay in payment. J Oper Res Soc 46:658-662

Balkhi ZT (2011) Optimal economic ordering policy with deteriorating items under different supplier trade credits for finite horizon case. Int J Prod Econ 133:216-223

Cárdenas-Barrón LE, Smith NR, Goyal SK (2010) Optimal order size to take advantage of a one-time discount offer with allowed backorders. Appl Math Model 34(6):1642-1652

Chang C-T, Ouyang L-Y, Teng J-T (2003) An EOQ model for deteriorating items under supplier credits linked to ordering quantity. Appl Math Model 27:983-996

Chang CT, Teng JT, Goyal SK (2008) Inventory lot-size models under trade credits: a review. Asia Pac J Oper Res 25:89-112

Chang CT, Teng JT, Chern MS (2010) Optimal manufacturer's optimal policies for deteriorating items in a supply chain with upstream and downstream trade credits. Int $\mathrm{J}$ Prod Econ 127:197-202

Chen LH, Kang FS (2010a) Integrated inventory models considering the two-level trade credit policy and price negotiation scheme. Eur J Oper Res 205:47-58

Chen LH, Kang FS (2010b) Coordination between vendor and buyer considering trade credit and items of imperfect quality. Int J Prod Econ 123:52-61

Chen S-H, Cárdenas-Barrón LE, Teng JT (2013a). Retailer's economic order quantity when the supplier offers conditionally 
permissible delay in payments link to order quantity. Int J Prod Econ. doi:10.1016/j.ijpe.2013.05.032 (in press)

Chen S-C, Chang C-T, Teng J-T (2013b) A comprehensive note on "Lot-sizing decisions for deteriorating items with two warehouses under an order-size-dependent trade credit". Int Trans Oper Res (in press)

Chern M-S, Pan Q, Teng J-T, Chan Y-L, Chen S-C (2013) Stackelberg solution in a vendor-buyer supply chain model with permissible delay in payments. Int $\mathrm{J}$ Prod Econ 144(1):397-404

Chung KJ, Cárdenas-Barrón LE (2013) The simplified solution procedure for deteriorating items under stock-dependent demand and two-level trade credit in the supply chain management. Appl Math Model 37(7):4653-4660

Chung KJ, Huang YF (2003) The optimal cycle time for EPQ inventory model under permissible delay in payments. Int J Prod Econ 84:307-318

Feng H, Li J, Zhao D (2013) Retailer's optimal replenishment and payment policies in the EPQ model under cash discount and twolevel trade credit policy. Appl Math Model 37:3322-3339

Goyal SK (1985) Economic order quantity under conditions of permissible delay in payments. J Oper Res Soc 36:335-338

Ho CH, Ouyang LY, Su CH (2008) Optimal pricing, shipment and payment policy for an integrated supplier-buyer inventory model with two-part trade credit. Eur J Oper Res 187:496-510

Hu F, Liu D (2010) Optimal replenishment policy for the EPQ model with permissible delay in payments and allowable shortages. Appl Math Model 34(10):3108-3117

Huang YF (2003) Optimal retailer's ordering policies in the EOQ model under trade credit financing. J Oper Res Soc 54:1011-1015

Huang YF (2007) Economic order quantity under conditionally permissible delay in payments. Eur J Oper Res 176:911-924

Huang YF, Hsu KH (2008) An EOQ model under retailer partial trade credit policy in supply chain. Int J Prod Econ 112:655-664

Hwang H, Shinn SW (1997) Retailer's pricing and lot sizing policy for exponentially deteriorating products under the condition of permissible delay in payments. Comput Oper Res 24:539-547

Jaggi CK, Goyal SK, Goel SK (2008) Retailer's optimal replenishment decisions with credit-linked demand under permissible delay in payments. Eur J Oper Res 190:130-135

Jaggi CK, Kapur PK, Goyal SK, Goel SK (2012) Optimal replenishment and credit policy in EOQ model under two-levels of trade credit policy when demand is influenced by credit period. Int $\mathbf{J}$ Syst Assur Eng Manag. doi:10.1007/s13198-012-0106-9

Jaggi CK, Goel SK, Mittal M (2013) Credit financing in economic ordering policies for defective items with allowable shortages. Appl Math Comput 219:5268-5282

Jamal AMM, Sarker BR, Wang S (1997) An ordering policy for deteriorating items with allowable shortage and permissible delay in payment. J Oper Res Soc 48:826-833

Liao J-J (2007) On an EPQ model for deteriorating items under permissible delay in payments. Appl Math Model 31(3):393-403

Liao JJ (2008) An EOQ model with noninstantaneous receipt and exponentially deteriorating items under two-level trade credit. Int J Prod Econ 113:852-861

Liao JJ, Huang KN, Chung KJ (2012) Lot-sizing decisions for deteriorating items with two warehouses under an order-sizedependent trade credit. Int J Prod Econ 137:102-115

Min J, Zhou YW, Zhao J (2010) An inventory model for deteriorating items under stock-dependent demand and two-level trade credit. Appl Math Model 34:3273-3285

Min J, Zhou YW, Liu G-Q, Wang S-D (2012) An EPQ model for deteriorating items with inventory-level-dependent demand and permissible delay in payments. Int J Syst Sci 43(6): 1039-1053
Ouyang L-Y, Chang C-T (2013) Optimal production lot with imperfect production process under permissible delay in payments and complete backlogging. Int J Prod Econ 144(2):610-617

Ouyang L-Y, Teng J-T, Chen L-H (2006) Optimal ordering policy for deteriorating items with partial backlogging under permissible delay in payments. J Global Optim 34:245-271

Ouyang L-Y, Yang C-T, Chan YL, Cárdenas-Barrón LE (2013) A comprehensive extension of the optimal replenishment decisions under two levels of trade credit policy depending on the order quantity. Appl Math Comput 224(1):268-277

Shah NH (1993) Probabilistic time-scheduling model for an exponentially decaying inventory when delay in payment is permissible. Int J Prod Econ 32:77-82

Skouri K, Konstantaras I, Papachristos S, Teng J-T (2011) Supply chain models for deteriorating products with ramp type demand rate under permissible delay in payments. Expert Syst Appl 38:14861-14869

Taleizadeh AA, Mohammadi B, Cárdenas-Barrón LE, Samimi H (2013) An EOQ model for perishable product with special sale and shortage. Int J Prod Econ 145(1):318-338

Teng JT (2002) On the economic order quantity under conditions of permissible delay in payments. J Oper Res Soc 53:915-918

Teng JT (2009) Optimal ordering policies for a retailer who offers distinct trade credits to its good and bad credit customers. Int $\mathbf{J}$ Prod Econ 119:415-423

Teng JT, Chang CT (2009) Optimal manufacturer's replenishment policies in the EPQ model under two-levels of trade credit policy. Eur J Oper Res 195:358-363

Teng JT, Goyal SK (2007) Optimal ordering policies for a retailer in a supply chain with up-stream and down-stream trade credits. J Oper Res Soc 58:1252-1255

Teng J-T, Krommyda IP, Skouri K, Lou K-R (2011) A comprehensive extension of optimal ordering policy for stock-dependent demand under progressive payment scheme. Eur J Oper Res 215:97-104

Teng J-T, Chang C-T, Chern M-S (2012a) Supply chain vendorbuyer inventory models with trade credit financing under an oncooperative and an integrated environments. Int J Syst Sci 43(11):2050-2061

Teng JT, Yang HL, Chern MS (2013) An inventory model for increasing demand under two levels of trade credit linked to order quantity. Appl Math Model 37:7624-7632

Teng J-T, Chen J, Goyal SK (2009) A comprehensive note on an inventory model under two levels of trade credit and limited storage space derived without derivatives. Appl Math Model 33:4388-4396

Teng J-T, Min J, Pan Q (2012b) Economic order quantity model with trade credit financing and non-decreasing demand. Omega 40:328-335

Thangam A (2012) Optimal price discounting and lot-sizing policies for perishable items in a supply chain under advance payment scheme and two-echelon trade credits. Int $\mathrm{J}$ Prod Econ 139:459-472

Thangam A, Uthayakumar R (2009) Two-echelon trade credit financing for perishable items in a supply chain when demand depends on both credit period and selling price. Comput Ind Eng 57:773-786

Thangam A, Uthayakumar R (2011) Two-echelon trade credit financing in a supply chain with perishable items and two different payment methods. Int J Oper Res 11(4):365-382

Tsao YC (2009) Retailers optimal ordering and discounting policies advance sales discount and trade credits. Comput Ind Eng 56:208-215

Tsao YC (2011) Replenishment policies considering trade credit and logistics risk. Scientia Iranica 18:753-758 
Tsao Y-C (2012) Determination of production runtime and warranty length under system maintenance and trade credits. Int J Syst Sci 43(12):2351-2360
Tsao YC, Sheen GJ (2012) A multi-item supply chain with credit periods and weight freight cost discounts. Int $\mathrm{J}$ Prod Econ 135:106-115 